\title{
Nonpositively curved metric in the positive cone of a finite von Neumann algebra*
}

\author{
Esteban Andruchow and Gabriel Larotonda ${ }^{\dagger}$
}

\begin{abstract}
In this paper we study the metric geometry of the space $\Sigma$ of positive invertible elements of a von Neumann algebra $\mathcal{A}$ with a finite, normal and faithful tracial state $\tau$. The trace induces an incomplete Riemannian metric $\langle x, y\rangle_{a}=\tau\left(y a^{-1} x a^{-1}\right)$, and though the techniques involved are quite different, the situation here resembles in many relevant aspects that of the $n \times n$ matrices when they are regarded as a symmetric space. For instance we prove that geodesics are the shortest paths for the metric induced, and that the geodesic distance is a convex function; we give an intrinsic (algebraic) characterization of the geodesically convex submanifolds $M$ of $\Sigma$, and under suitable hypothesis we prove a factorization theorem for elements in the algebra that resembles the Iwasawa decomposition for matrices. This factorization is obtained via a nonlinear orthogonal projection $\Pi_{M}: \Sigma \rightarrow M$, a map which turns out to be contractive for the geodesic distance. 1
\end{abstract}

\section{Introduction}

Let $\mathcal{A}$ be a von Neumann algebra with a finite (normal, faithful) trace $\tau$. Denote by $\mathcal{A}_{h}$ the set of selfadjoint elements of $\mathcal{A}$, by $G_{\mathcal{A}}$ the group of invertible elements, and by $\Sigma$ the set

$$
\Sigma=e^{\mathcal{A}_{h}}=\left\{a \in G_{\mathcal{A}}: a \geq 0\right\} ;
$$

$\Sigma$ is an open subset of $\mathcal{A}_{h}$ in the norm topology. Therefore if one regards it as a manifold, its tangent spaces identify with $\mathcal{A}_{h}$. We endow these tangent spaces with the (incomplete) Hilbert-Riemann metric

$$
<x, y>_{a}=\tau\left(x a^{-1} y a^{-1}\right), \quad a \in \Sigma, x, y \in \mathcal{A}_{h} .
$$

*2000 MSC. Primary 53C22, 58B20; Secondary 46L45.

${ }^{\dagger}$ Partially supported by IAM-CONICET.

${ }^{1}$ Keywords and phrases: weak Riemannian metric, minimizing geodesic, nonpositive curvature, convexity, normal projection, factorization 
Note that $\|x\|_{a}^{2}=<x, x>_{a}:=\tau\left(x a^{-1} x a^{-1}\right)$, and also that this metric is invariant for the action $I_{g}: x \mapsto g x g^{*}$, where $g \in G_{\mathcal{A}}$.

As in classical differential geometry, one obtains a metric $d$ for $\Sigma$ by considering

$$
\operatorname{dist}(a, b)=\inf \{\operatorname{Length}(\gamma): \gamma \text { is a smoooth curve joining } a \text { and } b\},
$$

where smooth means differentiable in the norm induced topology and the length of a curve $\gamma(t), t \in[0,1]$ is measured using the inner product above (1):

$$
\text { Length }(\gamma)=\int_{0}^{1}<\dot{\gamma}(t), \dot{\gamma}(t)>_{\gamma(t)}^{\frac{1}{2}} d t
$$

The purpose of this paper is the geometric study of the resulting metric space, and particularly, of its convex subsets.

If $\mathcal{A}$ is finite dimensional, i.e. a sum of matrix spaces, this metric is well known: it is the non positively curved Riemannian metric on the set of positive definite matrices, which is a universal model space for (finite dimensional) non positively curved manifolds on non compact type (see [4] and [5]).

If $\mathcal{A}$ is of type $\mathrm{II}_{1}$, the trace inner product is not complete, so that $\Sigma$, with the inner products $<,>_{a}$, is not a Hilbert-Riemann manifold properly speaking. For instance, the exponential map

$$
\exp : \mathcal{A}_{h} \rightarrow \Sigma, \quad \exp (x)=e^{x},
$$

which is a global diffeomorphism in the norm topology, is continuous but non differentiable in the 2-norm \|\|$_{2}$ induced by $\tau$ (namely $\|x\|_{2}=\tau\left(x^{*} x\right)^{\frac{1}{2}}$ ). The set $\Sigma$ itself is not a differentiable manifold with this norm.

However, the metric space $(\Sigma$, dist) behaves in many senses like in the finite dimensional setting. Let us mention a few issues:

1. The isometric action of the group $G_{\mathcal{A}}$ via $g \mapsto I_{g}$, where $I_{g}(x)=g x g^{*}$

2. Minimality of geodesics (i.e. solutions of Euler's equation are minimizing for the distance introduced above in (2), see Theorem 3.1)

3. Convexity of the map $t \mapsto \operatorname{dist}(\gamma(t), \delta(t))$ which gives distance among geodesics (Corollary 3.4)

4. Algebraic structure of (geodesically) convex subsets (Theorem 4.4).

5. Normal projections to convex submanifolds and their minimality (Lemma 5.3 and Theorem 5.4)

6. Existence and uniqueness of a factorization for invertible elements by means of convex submanifolds (Corollary [5.7) 


\section{Main inequalities}

The following inequality will be useful; its proof for $n \times n$ real matrices can be found in the inspiring paper of G.D. Mostow [5]. It is called by R. Bhatia [1] the exponential metric increasing property. Bhatia proves it for matrices (and for more general norms). However his proof for the 2-norm is valid almost verbatim in the infinite dimensional context for an arbitrary (finite, faithful) tracial state. We transcribe it. We use the fact that selfadjoint elements in a von Neumann algebra can be approximated by selfadjoint elements with finite spectrum.

Lemma 2.1. Let $\tau$ be a tracial faithful state in $\mathcal{A}$, and $x, y$ selfadjoint elements of $\mathcal{A}$. If exp denotes the usual exponential map, $\exp (x)=e^{x}$, then

$$
\|y\|_{2} \leq\left\|e^{-x} \operatorname{dexp}(y)\right\|_{2} .
$$

Proof. First we must establish the formula

$$
\operatorname{dexp}_{x}(y)=\int_{0}^{1} e^{t x} y e^{(1-t) x} d t
$$

Note that $\operatorname{dexp}_{x}(y)=\left.\frac{d}{d t} e^{x+t y}\right|_{t=0}$. Then

$$
\operatorname{dexp}_{x}(y)=y+\frac{1}{2}(y x+x y)+\frac{1}{6}\left(y x^{2}+x y x+x^{2} y\right)+\ldots
$$

On the other hand,

$$
e^{t x} y e^{(1-t) x}=y+t x y+(1-t) y x+\frac{1}{2}(1-t)^{2} y x^{2}+t(1-t) x y x+\frac{1}{2} t^{2} x^{2} y+\ldots
$$

Integrating this series (which is absolutely convergent) term by term proves the equality. Denote $a=e^{x}$. Let us show now that if $b$ is positive in $\mathcal{A}$,

$$
\left\|a^{\frac{1}{2}} b a^{\frac{1}{2}}\right\|_{2} \leq\left\|\int_{0}^{1} a^{t} b a^{1-t} d t\right\|_{2} .
$$

Assume first that $a$ has finite spectrum: $a=\sum_{i=1}^{n} \alpha_{i} p_{i}$, with $\alpha_{i}>0$ and $\sum_{i=1}^{n} p_{i}=$ 1. Then $a^{\frac{1}{2}} b a^{\frac{1}{2}}=\sum_{i, j=1}^{n} \alpha_{i}^{\frac{1}{2}} \alpha_{j}^{\frac{1}{2}} p_{i} b p_{j}$. Therefore

$$
\left\|a^{\frac{1}{2}} b a^{\frac{1}{2}}\right\|_{2}^{2}=\sum_{i, j=1}^{n} \alpha_{i} \alpha_{j} \tau\left(p_{i} b p_{j} b p_{i}\right)
$$

Analogously $\int_{0}^{1} a^{t} b a^{1-t} d t=\sum_{i, j}^{n} \int_{0}^{1} \alpha_{i}^{t} \alpha_{j}^{1-t} d t p_{i} b p_{j}$ and

$\left\|\int_{0}^{1} a^{t} b a^{1-t} d t\right\|_{2}^{2}=\sum_{i, j}^{n} \int_{0}^{1} \alpha_{i}^{2 t} \alpha_{j}^{2(1-t)} d t \tau\left(p_{i} b p_{j} b p_{i}\right)=\sum_{i, j}^{n} \frac{\alpha_{i}^{2}-\alpha_{j}^{2}}{2 \ln \alpha_{i}-2 \ln \alpha_{j}} \tau\left(p_{i} b p_{j} b p_{i}\right)$. 
Note that $p_{i} b p_{j} b p_{i}$ is positive. Also one has the elementary inequality

$$
\sqrt{s t} \leq \frac{s-t}{\ln s-\ln t}
$$

for $s, t>0$. Then

$$
\alpha_{i} \alpha_{j} p_{i} b p_{j} b p_{i} \leq \frac{\alpha_{i}^{2}-\alpha_{j}^{2}}{2 \ln \alpha_{i}-2 \ln \alpha_{j}} p_{i} b p_{j} b p_{i}
$$

Taking traces and adding yields (4) in this case. In the general case, the inequality follows by approximating (in norm) the element $a$ with a positive elements with finite spectrum.

As in [1], put $b=e^{-x / 2} y e^{-x / 2}$ in (4):

$$
\begin{aligned}
\|y\|_{2} \leq & \left\|\int_{0}^{1} e^{t x}\left(e^{-x / 2} y e^{-x / 2}\right) e^{(1-t) x} d t\right\|_{2}=\left\|e^{-x / 2} \int_{0}^{1} e^{t x} y e^{(1-t) x} d t e^{-x / 2}\right\|_{2} \\
& =\left\|e^{-x / 2}\left(\operatorname{dexp}_{x}(y)\right) e^{-x / 2}\right\|_{2} .
\end{aligned}
$$

If $a$ is positive and invertible and $b$ is selfadjoint, by the Cauchy-Schwarz inequality for $\tau$, one has

$$
\left\|a^{-\frac{1}{2}} b a^{-\frac{1}{2}}\right\|_{2}^{2}=\tau\left(a^{-1} b a^{-1} b\right) \leq \tau\left(a^{-1} b^{2} a^{-1}\right)^{\frac{1}{2}} \tau\left(b a^{-2} b\right)^{\frac{1}{2}}=\left\|a^{-1} b\right\|_{2}^{2} .
$$

Using this inequality for $a=e^{x}$ and $b=\operatorname{dexp}_{x}(y)$ one obtains

$$
\|y\|_{2} \leq\left\|e^{-x / 2}\left(\operatorname{dexp}_{x}(y)\right) e^{-x / 2}\right\|_{2} \leq\left\|e^{-x}(\operatorname{dexp} x(y))\right\|_{2} .
$$

Corollary 2.2. For any $x \in \mathcal{A}_{h}$, the map $T_{x}: y \mapsto e^{-x / 2} \operatorname{dexp} p_{x}(y) e^{-x / 2}$ is bounded, symmetric for the 2-inner product (when restricted to $\mathcal{A}_{h}$ ) and invertible. The inverse is contractive i.e $T_{x}^{-1}(z)\left\|_{2} \leq\right\| z \|_{2}$.

Proof. The map is clearly bounded and invertible, the bound for the inverse follows from the proof of the previous Lemma. To prove that it is symmetric, note that

$$
\begin{gathered}
<T_{x}(y), z>{ }_{2}=\tau\left(z T_{x}(y)\right)=\tau\left(e^{-x / 2} \sum_{n \geq 0} \frac{1}{n !} \sum_{p+q=n-1} x^{p} y x^{q} e^{-x / 2} z\right)= \\
=\sum_{n \geq 0} \frac{1}{n !} \sum_{p+q=n-1} \tau\left(e^{-x / 2} x^{p} y x^{q} e^{-x / 2} z\right)=\sum_{n \geq 0} \frac{1}{n !} \sum_{p+q=n-1} \tau\left(x^{p} e^{-x / 2} y e^{-x / 2} x^{q} z\right)= \\
=\sum_{n \geq 0} \frac{1}{n !} \sum_{p+q=n-1} \tau\left(e^{-x / 2} x^{q} z x^{p} e^{-x / 2} y\right)=\tau\left(T_{x}(z) y\right)=<y, T_{x}(z)>_{2} .
\end{gathered}
$$




\section{Geodesic distance}

For $X, Y$ smooth vector fields in $\Sigma$ and $p \in \Sigma$, we introduce the expression

$$
\left(\nabla_{X} Y\right)_{p}=\{X(Y)\}_{p}-\frac{1}{2}\left(X_{p} p^{-1} Y_{p}+Y_{p} p^{-1} X_{p}\right)
$$

where $X(Y)$ denotes derivation of the vector field $Y$ in the direction of $X$ (performed in the linear space $\mathcal{A}_{h}$ ). Note that $\nabla$ is clearly symmetric and verifies all the formal identities of a connection. The compatibility condition between the connection and the metric

$$
\frac{d}{d t}<X, Y>_{\gamma}=<\nabla_{\dot{\gamma}} X, Y>_{\gamma}+<X, \nabla_{\dot{\gamma}} Y>_{\gamma}
$$

is fulfilled for any smooth curve $\gamma \subset \Sigma$ and $X, Y$ tangent vector fields along $\gamma$. This identity is straightforward from the definitions for both terms and the cyclicity of the trace. This says that $\nabla$ is the "Levi-Civita" connection of the metric introduced. Euler's equation $\nabla_{\dot{\gamma}} \dot{\gamma}=0$ reads $\ddot{\gamma}=\dot{\gamma} \gamma^{-1} \dot{\gamma}$, and it is easy to see that the (unique) solution of this equation with $\gamma(0)=p, \gamma(1)=q$ is given by the curve

$$
\delta_{p q}(t)=p^{\frac{1}{2}}\left(p^{-\frac{1}{2}} q p^{-\frac{1}{2}}\right)^{t} p^{\frac{1}{2}} .
$$

Note that $\delta_{p q} \subset \Sigma$ because $a b a$ is positive invertible whenever $a, b$ are positive invertible.

We will prove that the shortest path joining $p$ to $q$ is given by the formula above (Theorem 13.1); these curves look formally equal to the geodesics between positive definite matrices (regarded as a symmetric space).

We will use $\operatorname{Exp}_{p}$ to denote the exponential map of $\Sigma$. Note that

$$
\operatorname{Exp}_{p}(v)=p^{\frac{1}{2}} \mathrm{e}^{p^{-\frac{1}{2}} v p^{-\frac{1}{2}}} p^{\frac{1}{2}}
$$

Rearranging the exponential series we get a simpler expression

$$
\operatorname{Exp}_{p}(v)=p \mathrm{e}^{p^{-1} v}=\mathrm{e}^{v p^{-1}} p .
$$

A straightforward computation also shows that for $p, q \in \Sigma$ we have

$$
\operatorname{Exp}_{p}^{-1}(q)=p^{\frac{1}{2}} \ln \left(p^{-\frac{1}{2}} q p^{-\frac{1}{2}}\right) p^{\frac{1}{2}} .
$$

As mentioned in the introduction, we measure curves in $\Sigma$ using the norms in the tangent space, namely

$$
\operatorname{Length}(\alpha)=\int_{0}^{1}\|\dot{\alpha}(t)\|_{\alpha(t)} d t
$$

We have $\left\|\delta_{p, q}^{\cdot}(t)\right\|_{\delta_{p, q}(t)} \equiv\left\|\ln \left(p^{-\frac{1}{2}} q p^{-\frac{1}{2}}\right)\right\|_{2}$, so for the geodesics introduced in equation (6), we have $L\left(\delta_{p, q}\right)=\left\|\ln \left(p^{-\frac{1}{2}} q p^{-\frac{1}{2}}\right)\right\|_{2}$. 
Theorem 3.1. Let $a, b \in \Sigma$. Then the geodesic $\delta_{a, b}$ is the shortest curve joining a and $b$ in $\Sigma$, if the length of curves is measured with the metric defined above.

Proof. Let $\gamma$ be a smooth curve in $\Sigma$ with $\gamma(0)=a$ and $\gamma(1)=b$. We must compare the length of $\gamma$ with the length of $\delta_{a, b}$. Since the invertible group acts isometrically for the metric, it preserves the lengths of curves. Thus we way act with $a^{-\frac{1}{2}}$, and suppose that both curves start at 1 , or equivalently, $a=1$. Therefore $\delta_{1, b}(t)=\delta(t)=e^{t x}$, with $x=\ln b$. The length of $\delta$ is therefore $\tau\left(x^{2}\right)^{\frac{1}{2}}=\|x\|_{2}$. The proof follows easily from the inequality proved above. Indeed, since $\gamma$ is a smooth curve in $\Sigma$, it is of the form $\gamma(t)=e^{\alpha(t)}$, with $\alpha=\ln \gamma$. Then $\alpha$ is a smooth curve of selfadjoints with $\alpha(0)=0$ and $\alpha(1)=x$. Moreover,

$$
\tau\left(\left(\gamma^{-1} \dot{\gamma} \gamma^{-1} \dot{\gamma}\right)^{\frac{1}{2}}=\left\|e^{-\alpha} e^{\dot{\alpha}}\right\|_{2}=\left\|e^{-\alpha} \operatorname{dexp}(\dot{\alpha})\right\|_{2} .\right.
$$

By the inequality in the above lemma, this is not smaller than $\|\dot{\alpha}\|_{2}$. Then

$$
\int_{0}^{1} \tau\left(\left(\gamma^{-1} \dot{\gamma} \gamma^{-1} \dot{\gamma}\right)^{\frac{1}{2}} d t \geq \int_{0}^{1}\|\dot{\alpha}\|_{2} d t \geq\left\|\int_{0}^{1} \dot{\alpha} d t\right\|_{2}=\|x\|_{2}=\tau\left(x^{2}\right)^{\frac{1}{2}} .\right.
$$

Remark 3.2. The geodesic distance induced by the metric is given by

$$
\operatorname{dist}(a, b)=\tau\left(\ln \left(a^{-\frac{1}{2}} b a^{-\frac{1}{2}}\right)^{2}\right)^{\frac{1}{2}} .
$$

The curvature tensor [2] is given by

$$
R_{a}(x, y) z=-\frac{1}{4} a\left[\left[a^{-1} x, a^{-1} y\right], a^{-1}\right]
$$

where $[$,$] is the usual commutator, i.e [x, y]=x y-y x$.

Let $J(t)$ be a Jacobi field along a geodesic $\delta$ of $\Sigma$. That is, $J$ is a solution of the differential equation

$$
\frac{D^{2} J}{d t^{2}}+R_{\delta}(J, \dot{\delta}) \dot{\delta}=0
$$

Next we show that the norm of a Jacobi field is convex. If $x, y \in \mathcal{A}_{h}$ are regarded as tangent vectors of $\Sigma$ at the point $a$, then the following condition (which is a non positive sectional curvature condition) holds:

$$
<R_{a}(x, y) y, x>_{a}=\tau\left(R_{a}(x, y) y a^{-1} x a^{-1}\right) \leq 0 .
$$

The proof of this fact is straightforward. Then

$$
\begin{aligned}
\frac{d^{2}}{d t^{2}}<J, J>_{\gamma} & =2\left\{<\frac{D^{2} J}{d t^{2}}, J>_{\gamma}+<\frac{D J}{d t}, \frac{D J}{d t}>_{\gamma}\right\}= \\
& =2\left\{-<R_{\gamma}(J, \dot{\gamma}) \dot{\gamma}, J>_{\gamma}+<\frac{D J}{d t}, \frac{D J}{d t}>_{\gamma}\right\} \geq 0 .
\end{aligned}
$$

In other words, the smooth function $t \mapsto\langle J, J\rangle_{\gamma}$ is convex. We shall need convexity of the norm of the Jacobi field (and not of the square of the norm just noted). 
Proposition 3.3. Let $\gamma$ be a geodesic of $\Sigma$ and $J$ a Jacobi field along $\gamma$. The real map $t \mapsto<J, J>\frac{1}{2}$ is convex.

Proof. Clearly, is suffices to prove this assertion for a field $J$ which does not vanish. As in Theorem 1 of [3], by the invariance of the connection and the metric under the action of $G_{\mathcal{A}}$, it suffices to consider the case of a geodesic $\gamma(t)=e^{t x}$ starting at $1 \in \Sigma\left(x \in \mathcal{A}_{h}\right)$. For the field $K(t)=e^{-t x / 2} J(t) e^{-t x / 2}$ the Jacobi equation translates into

$$
4 \ddot{K}=K x^{2}+x^{2} K-2 x K x .
$$

Moreover

$$
<J, J>\frac{1}{2}=\tau\left(\gamma^{-1} J \gamma^{-1} J\right)^{\frac{1}{2}}=\tau\left(K^{2}\right)^{\frac{1}{2}}=\|K\|_{2} .
$$

Let us prove therefore that the map $t \mapsto f(t)=\|K(t)\|_{2}$ is convex, for any (non vanishing) solution $K$ of (8). Note that $f(t)$ is smooth, and $\dot{f}=\tau\left(K^{2}\right)^{-\frac{1}{2}} \tau(K \dot{K})$. Then

$$
\ddot{f}=-\tau\left(K^{2}\right)^{-\frac{3}{2}} \tau(K \dot{K})^{2}+\tau\left(K^{2}\right)^{-\frac{1}{2}}\left\{\tau\left(\dot{K}^{2}\right)+\tau(K \ddot{K})\right\} .
$$

Let us multiply this expresion by $\tau\left(K^{2}\right)^{\frac{3}{2}}$ to obtain

$$
-\tau(K \dot{K})^{2}+\tau\left(K^{2}\right) \tau\left(\dot{K}^{2}\right)+\tau\left(K^{2}\right) \tau(K \ddot{K}) .
$$

The first two terms add up to a non negative number. Indeed, one has $\tau(K \dot{K})^{2} \leq$ $\tau\left(K^{2}\right) \tau\left(\dot{K}^{2}\right)$ by the Cauchy-Schwarz inequality for the trace $\tau$. Let us examine the third term $\tau\left(K^{2}\right) \tau(K \ddot{K})$. It suffices to show that $\tau(K \ddot{K})$ is non negative. Using (8),

$$
\tau(K \ddot{K})=\frac{1}{4}\left\{\tau\left(K^{2} x^{2}\right)+\tau\left(K x^{2} K\right)-2 \tau(K x K x)\right\}=\frac{1}{2}\left\{\tau\left(K^{2} x^{2}\right)-\tau(K x K x)\right\} .
$$

This number is positive, again by the Cauchy-Schwarz inequality:

$$
\tau(K x K x)=\tau\left((x K)^{*} K x\right) \leq \tau\left((x K)^{*} x K\right)^{\frac{1}{2}} \tau\left((K x)^{*} K x\right)^{\frac{1}{2}}=\tau\left(K^{2} x^{2}\right) .
$$

Corollary 3.4. If $\gamma$ and $\delta$ are geodesics, the map $f(t)=\operatorname{dist}(\gamma(t), \delta(t))$ is a convex function of $t$.

Proof. As in Theorem 2 of [3, distance between $\gamma(t)$ and $\delta(t)$ is given by the geodesic $\alpha_{t}(s)$ obtained moving the $s$ variable in a geodesic square $h(s, t)$ with vertices $\gamma\left(t_{0}\right), \delta\left(t_{0}\right), \gamma\left(t_{1}\right), \delta\left(t_{1}\right)$. Taking the partial derivative along the $s$ direction gives a Jacobi field $J(s, t)$ along the geodesic $\beta_{s}(t)=h(s, t)$ and it also gives the speed of $\alpha_{t}$. Hence

$$
f(t)=\int_{0}^{1}\left\|\frac{\partial \alpha_{t}}{\partial s}(s)\right\|_{\alpha_{t}(s)} d s=\int_{0}^{1}\|J(s, t)\|_{h(s, t)} d s .
$$

This equation says that $f(t)$ can be written as the limit of a convex combination of convex functions $u_{i}(t)=\left\|J\left(s_{i}, t\right)\right\|_{h\left(s_{i}, t\right)}$, so $f$ must be convex itself. 
Lemma 3.5. The following inequality holds for any $x, y \in \mathcal{A}_{h}$ :

$$
\operatorname{dist}\left(e^{x}, e^{y}\right)=\left\|\ln \left(e^{x / 2} e^{-y} e^{x / 2}\right)\right\|_{2} \geq\|x-y\|_{2} .
$$

Proof. Take $\gamma(t)=e^{t x}, \delta(t)=e^{t y}$ and $f$ as in the previous corollary. Note that $f(0)=0$, hence $f(t) / t \leq f(1)$ for any $0<t \leq 1$; hence $\lim _{t \rightarrow 0^{+}} f(t) / t \leq f(1)$. Now note that

$$
f(t) / t=\frac{1}{t}\left\|\ln \left(e^{t x / 2} e^{-t y} e^{t x / 2}\right)\right\|_{2}=\tau\left(\left[\frac{1}{t} \ln \left(e^{t x / 2} e^{-t y} e^{t x / 2}\right)\right]^{2}\right)^{\frac{1}{2}} .
$$

Since $\lim _{t \rightarrow 0^{+}} \frac{1}{t} \ln \left(e^{t x / 2} e^{-t y} e^{t x / 2}\right)=\left.\frac{d}{d t}\right|_{t=0} \ln \left(e^{t x / 2} e^{-t y} e^{t x / 2}\right)$, and the logarithm of $\beta(t)=e^{t x / 2} e^{-t y} e^{t x / 2}$ can be approximated uniformly by polinomials $p_{n}(\beta)=$ $\sum_{k} \alpha_{n, k} \beta^{k}$ for $t$ close enough to zero (note that $\beta(0)=1$ ), and $\left.\frac{d}{d t} \beta\right|_{t=0}=x-y$, we have the desired inequality.

Using the inner product in each tangent space, we can talk about angles between curves and more general subsets of $\Sigma$ in a natural way; in particular we have:

Lemma 3.6. The sum of the inner angles of any geodesic triangle in the manifold $\Sigma$ is less or equal than $\pi$

Proof. Squaring both sides of inequality (9) leads (by the invariance of the metric for the action of $G_{\mathcal{A}}$ ) to

$$
l_{i}^{2} \geq l_{i+1}^{2}+l_{i-1}^{2}-2 l_{i+1} l_{i-1} \cos \left(\alpha_{i}\right)
$$

where $l_{i}$ are the sides of any geodesic triangle and $\alpha_{i}$ is the angle opposite to $l_{i}$. These inequalities say that we can construct an Euclidian comparison triangle in the affine plane with sides $l_{i}$; they also say that the angle $\beta_{i}$ (opposite to $l_{i}$ for this flat triangle) is bigger than $\alpha_{i}$. Adding the three angles we have $\alpha_{1}+\alpha_{2}+\alpha_{3} \leq$ $\beta_{1}+\beta_{2}+\beta_{3}=\pi$.

\section{Convex sets}

We are interested in the convex subsets of $\Sigma$, that is, subsets $M \subset \Sigma$ such that the ambient geodesic joining two points in $M$ stays in $M$ for any value of $t$. Note that the simplest of such objects are the geodesics.

It's not hard to see that when two elements $a, b \in \Sigma$ commute, the geodesic triangle spanned by $a, b$ and 1 is convex, hence there is a flat surface containing $a, b$ and 1 ; indeed, the triangle in $\Sigma$ is the image of the plane triangle with vertices $0, e_{1}, e_{2}$ under the map $T: \mathbb{R}^{2} \rightarrow \Sigma$ given by

$$
T(x, y)=e^{x \ln (a)-y \ln (b)}
$$


In particular the geodesic joining $a$ and $b$ is the image of the segment

$$
\{(x, y): x, y \geq 0, x+y=1\} .
$$

This is not true in the general case (though the length of the segment is a lower bound for the length of that geodesic, as Lemma 9 shows).

Definition 4.1. An exponential set $M \subset \Sigma$ is the exponential of a (closed, selfadjoint) subspace through the origin. In other words, $M=e^{H}$ with $H$ a closed subspace of $\mathcal{A}_{h}$.

Lemma 4.2. If $M$ is a convex exponential set in $\Sigma$, the geodesic symmetry $\sigma_{p}$ : $q \mapsto p q^{-1} p$ maps $M$ into $M$ for any $p \in M$

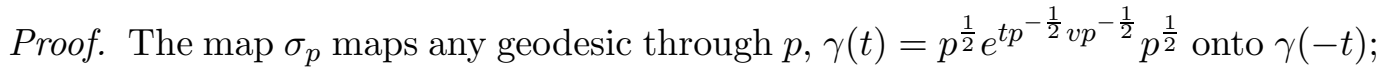
now it is clear that it is an isommetry of $\Sigma$ and it maps $M$ into $M$.

Note that, if $M$ is convex and $a \in M$, then $a^{\alpha}=e^{\alpha \ln a}$ is in $M$ for any real $\alpha$. This observation together with the previous lemma leads to the following characterization of convexity:

Proposition 4.3. If $M$ is a convex, exponential set in $\Sigma$, then

$$
a b a \in M \text { whenever } a, b \in M
$$

Proof. Note that $a b a=a^{\frac{3}{2}}\left(a^{\frac{1}{2}} b^{-1} a^{\frac{1}{2}}\right)^{-1} a^{\frac{3}{2}}=\sigma_{a^{\frac{3}{2}}} \circ \sigma_{a^{\frac{1}{2}}}(b)$.

The converse of this last statement is also true (this can be easily seen iterating the property above in order to construct $\gamma(t)$ for given $p, q \in M)$.

Let us see how this property looks in the tangent $H$ (recall that $M=e^{H}$ ). This result is related to the results of [7] by $\mathrm{H}$. Porta and L. Recht:

Theorem 4.4. If $H$ is a closed subspace of $\mathcal{A}_{h}$ (in the norm topology of $\mathcal{A}$ ), then $M=e^{H}$ is a geodesically convex subset of $\Sigma$ if and only if $[x,[x, y]] \in H$ for any $x, y \in H$.

Proof. We use property (10) above to identify convex sets; the proof follows the guidelines of [5] for matrices, and we translate it here.

We first assume $H$ has the double bracket property. Set $D_{x}: \mathcal{A} \rightarrow \mathcal{A}, D_{x}=L_{x}-R_{x}$, the difference between left and right multiplication by $x$ in $\mathcal{A}$.

Let's consider the completion of $\mathcal{A}$ with respect to the trace, namely $\mathcal{H}=L^{2}(\mathcal{A}, \tau)$. Clearly $\mathcal{H}_{\mathbb{R}}=L_{\mathbb{R}}^{2}\left(\mathcal{A}_{h}, \tau\right)$ contains as a proper, closed subspace the completion of $H$, namely $\mathcal{H}_{1}=L_{\mathbb{R}}^{2}(H, \tau)$. Since $\tau$ is a normal trace, the involution * extends to a bounded antilinear operator $J$ of $\mathcal{H}_{\mathbb{R}}$, and the map $D_{x}$ extends uniquely to a bounded linear operator of $\mathcal{H}_{\mathbb{R}}$ (which we will still call $D_{x}$ ). 
First we establish the identity

$$
T_{x}(y)=g\left(D_{x} / 2\right)(y)
$$

where $T_{x}$ is the extension of the map from Corollary 2.2, and

$$
g(z):=\frac{\sinh (z)}{z}=\sum_{n \geq 0} \frac{z^{2 n}}{(2 n+1) !}
$$

is an entire function. Note that $g(z)=(2 z)^{-1}\left(e^{z}-e^{-z}\right)$. To prove (11), we take derivative with respect to $t$ in the identity $X(t) e^{X(t)}=e^{X(t)} X(t)$, where $X(t)=$ $x+t y$; after rearranging the terms we come up with

$$
\frac{1}{2}\left(e^{D_{x} / 2}-e^{-D_{x} / 2}\right) y=\left(D_{x} \circ T_{x}\right)(y) .
$$

Note that if $D_{x}$ were invertible, we would be set; this is not necessarily the case. However, $D_{x}^{2}=D_{x} \circ D_{x}$ is selfadjoint when restricted to $\mathcal{A}$, and since $T_{x}$ (more precisely, its extension) is also sefaldjoint (Corollary 2.2), the operator $T=T_{x}-$ $g\left(D_{x} / 2\right)$ is selfadjoint on $\mathcal{A}$, and hence on $\mathcal{H}$ (note that $g(z)$ involves only even powers of $z$ ). The equation above says that we have proved that $\left(D_{x} \circ T\right)(y)=0$ for any $y \in \mathcal{A}$; in other words $T$ maps $\mathcal{H}$ into $\{x\}^{\prime}=\overline{\left\{b \in \mathcal{A}_{h}: b x=x b\right\}}$. A straightforward computation shows that $T b=0$ for any $b \in\{x\}^{\prime}$, which proves that $T=0$, i.e. equation (11) holds.

Now, for $x, y \in H$ consider the curve $e^{\alpha(t)}=e^{t x} e^{y} e^{t x}$. Clearly $\alpha(0)=y \in H \subset \mathcal{H}_{1}$; we will prove that $\alpha$ obeys a differential equation in $\mathcal{H}_{\mathbb{R}}$ which has a flow that maps $\mathcal{H}_{1}$ into $\mathcal{H}_{1}$, and by the uniqueness of the solution of such equation we will have $e^{x} e^{y} e^{x}=e^{\alpha(1)} \in e^{H}=M$.

Differentiating at $t=t_{0}$ the equation yields to

$$
\begin{aligned}
x e^{\alpha\left(t_{0}\right)}+e^{\alpha\left(t_{0}\right)} x & =\operatorname{dexp}_{\alpha\left(t_{0}\right)}\left(\dot{\alpha}\left(t_{0}\right)\right)=e^{\alpha\left(t_{0}\right) / 2} T_{\alpha\left(t_{0}\right)}\left(\dot{\alpha}\left(t_{0}\right)\right) e^{\alpha\left(t_{0}\right) / 2}= \\
& =e^{\alpha\left(t_{0}\right) / 2} g\left(D_{\alpha\left(t_{0}\right)} / 2\right)\left(\dot{\alpha}\left(t_{0}\right)\right) e^{\alpha\left(t_{0}\right) / 2} .
\end{aligned}
$$

Note that $g(z)$ is invertible whenever $z$ is a bounded linear operator, and also that the power series for $z \operatorname{coth}(z / 2)$ involves only even powers of $z$. On the other hand, $D_{z} / 2=D_{z / 2}$ and $e^{z} x e^{-z}=e^{D_{z}} x$, hence

$$
\begin{aligned}
\dot{\alpha} & =g^{-1}\left(D_{\alpha} / 2\right) \circ\left(e^{-\alpha / 2} x e^{\alpha / 2}+e^{\alpha / 2} x e^{-\alpha / 2}\right)= \\
& =g^{-1}\left(D_{\alpha / 2}\right) \circ\left(e^{D_{\alpha} / 2}+e^{-D_{\alpha} / 2}\right)(x)=D_{\alpha} \operatorname{coth}\left(D_{\alpha / 2}\right)(x)=\sum_{n} c_{n} D_{\alpha}^{2 n} x= \\
& =\sum_{n} c_{n} D_{\alpha}^{2} \circ \cdots \circ D_{\alpha}^{2}(x)=F(\alpha) .
\end{aligned}
$$

Since $D_{z}^{2}(x)=[z,[z, x]], F(z)=\sum_{n} c_{n} D_{z}^{2 n}(x)$ can be regarded as a map from $\mathcal{H}_{1}$ to $\mathcal{H}_{1}$, and since it is clearly an analytic map of $\mathcal{H}$ into $\mathcal{H}$, it fulfills a Lipschitz 
condition. Now the unique solution must be $\alpha(t)=\ln \left(e^{t x} e^{y} e^{t x}\right)$. This proves that $M$ is convex whenever $H$ has the double bracket property.

To prove the other implication, assume $M=e^{H}$ is convex and $H$ is closed in the norm topology of $\mathcal{A}_{h}$. Clearly the path $\alpha$ stays in $H$ for any value of $t$ (here $\left.e^{\alpha(t)}=e^{t x} e^{y} e^{t x}\right)$, and the same is true for $\dot{\alpha}$. Now since $\dot{\alpha}(t)=D_{\alpha(t)} \operatorname{coth}\left(D_{\alpha(t)} / 2\right) x$, we have

$$
\lim _{t \rightarrow 0} \frac{\dot{\alpha}(t)-\dot{\alpha}(0)}{t^{2}}=\lim _{t \rightarrow 0} \frac{\left(1+\frac{1}{12} t^{2} D_{\alpha(t)}^{2}\right) x-x}{t^{2}}+t O(t)=\frac{1}{12} D_{y}^{2}(x)
$$

which proves that $D_{y}^{2}(x)=[y,[y, x]]$ belongs to $H$ whenever $x$ and $y$ are in $H$.

\section{Projections}

¿From now on assume $M=e^{H}$ is a convex exponential set in $\Sigma$. As before, we identify the derivatives of all the geodesics at $p \in M$ with the tangent space of $M$ at $p$, in order to define the angles between curves and sets in a natural way: note that in this way, $T_{1} M=H$ and $T_{p} M=p^{\frac{1}{2}} H p^{\frac{1}{2}}$ (which can be thought of as the parallel transport along the geodesic joining 1 and $p$ in $M$ ).

In particular, $T_{1} \Sigma$ is naturally identified with $\mathcal{A}_{h}$ and the same is true for $T_{p} \Sigma$, for any $p \in \Sigma$, since $p^{\frac{1}{2}} \mathcal{A}_{h} p^{\frac{1}{2}}=\mathcal{A}_{h}$ (this is clear also from the fact that $\Sigma$ is open in $\left.\mathcal{A}_{h}\right)$.

Lemma 5.1. Let $r \in \Sigma$. There is at most one point $p=\Pi_{M}(r)$ in $M$ such that the geodesic joining $r$ and $p$ is orthogonal to $M$ at $p$

Proof. Assume there are two points $p$ and $p^{\prime}$ in $M$ and two vectors $v$ and $v^{\prime}$ orthogonal to $M$ at $p$ and $p^{\prime}$ respectively such that $\gamma_{1}(1)=\operatorname{Exp}_{p}(v)=\gamma_{2}(1)=\operatorname{Exp}_{p^{\prime}}\left(v^{\prime}\right):=$ $r$, and consider the geodesic triangle with sides the given geodesics and the unique geodesic in $M$ joining $p$ and $p^{\prime}$. Since the angles at $p$ and $p^{\prime}$ are right angles, and the summ of the inner angles of any such geodesic triangle is less or equal than $\pi$, it must be that the angle at $r$ is zero: since geodesics are unique (given an initial velocity and an initial position $r$ ), it must be that $\gamma_{1}=\gamma_{2}$, hence $p=p^{\prime}$ and $v=v^{\prime}$.

Set $N M$ as the normal bundle of $M$, i.e. $N M=\left\{(p, v): p \in M, v \in\left(T_{p} M\right)^{\perp}\right\}$.

Consider the map $E: N M \rightarrow \Sigma$ given by $(p, v) \mapsto \operatorname{Exp}_{p}(v)$; since $E$ is analytic and with the right identifications has differential (at $(p, 0))$ the identity map, $E(N M)$ contains an open neighbourhood of $M$ in $\Sigma$ (with the norm topology).

Lemma 5.2. The map $\Pi_{M}: E(N M) \rightarrow M$ that assigns the endpoint of the minimizing geodesic is contractive for the geodesic metric. 
Proof. If $r, s$ are two points in $E(N M)$, we will prove that this projection is contractive. Assume $\Pi_{M}(r)=p, \Pi_{M}(s)=q \in M, v \in T_{p} \Sigma$ is orthogonal to $M$ at $p$ and $w$ is orthogonal to $M$ at $q$; let's consider the distance function

$$
f(t)=\operatorname{dist}^{2}\left(\operatorname{Exp}_{p}(t v), \operatorname{Exp}_{q}(t w)\right)=\operatorname{dist}^{2}\left(\gamma_{1}(t), \gamma_{2}(t)\right)
$$

where $\gamma_{1}(t)$ is the only geodesic with initial velocity $v$ starting at $p$ and $\gamma_{2}$ is the only geodesic with initial speed $w$ starting at $q$. Namely,

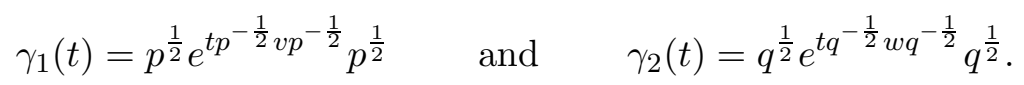

Since $v \in\left(T_{p} M\right)^{\perp}$ and $w \in\left(T_{q} M\right)^{\perp}$, we have

$$
\begin{aligned}
& <v, p^{\frac{1}{2}} x p^{\frac{1}{2}}>_{p}=\tau\left(x p^{-\frac{1}{2}} v p^{-\frac{1}{2}}\right)=0 \text { for any } x \in H=T_{1} M \text { and } \\
& <w, q^{\frac{1}{2}} y q^{\frac{1}{2}}>_{q}=\tau\left(y q^{-\frac{1}{2}} w q^{-\frac{1}{2}}\right)=0 \text { for any } y \in H=T_{1} M .
\end{aligned}
$$

Now we use the formula $\operatorname{dist}\left(e^{A}, e^{B}\right)=\left\|\ln \left(e^{A / 2} e^{-B} e^{A / 2}\right)\right\|_{2}$ for $A=\ln \left(\gamma_{1}(t)\right)$ and $B=\ln \left(\gamma_{2}(t)\right)$, to write

$$
f(t)=\left\|\ln \left(\gamma_{1}^{\frac{1}{2}} \gamma_{2}^{-1} \gamma_{1}^{\frac{1}{2}}\right)\right\|_{2}^{2}=\tau\left(\ln ^{2}\left(\gamma_{1}^{\frac{1}{2}} \gamma_{2}^{-1} \gamma_{1}^{\frac{1}{2}}\right)\right) .
$$

Assume that $C$ is a simple, positively oriented curve in $\mathbb{C}$, around the spectrum of $\alpha_{0}=p^{\frac{1}{2}} q^{-1} p^{\frac{1}{2}}$. Then we can use the Cauchy formula to calculate $\ln ^{2}(a)$ for any element $a \in \mathcal{A}$ such that $\sigma(a) \subset \operatorname{int}(C)$, namely

$$
\ln ^{2}(a)=\frac{1}{2 \pi i} \int_{C} \ln ^{2}(z)(z-a)^{-1} d z .
$$

Naming $\alpha(t)=\gamma_{1}^{\frac{1}{2}}(t) \gamma_{2}^{-1}(t) \gamma_{1}^{\frac{1}{2}}(t)$, this formula holds true for $\alpha_{0}=\alpha(0)$ and for $\alpha(t)$ for $t$ sufficiently small. Note that

$$
f(t)=\tau\left(\gamma^{-\frac{1}{2}}(t) \gamma^{\frac{1}{2}}(t) \ln ^{2}(\alpha(t))\right)=\tau\left(\gamma^{-\frac{1}{2}}(t) \ln ^{2}(\alpha(t)) \gamma^{\frac{1}{2}}(t)\right) .
$$

If $x$ is invertible in $\mathcal{A}, x g(a) x^{-1}=g\left(x a x^{-1}\right)$ for any element $a \in \mathcal{A}$ and any analytic function $g$ in a neighbourhood of $\sigma(a)$. Then

$$
f(t)=\tau\left(\ln ^{2}\left[\gamma_{1}(t) \gamma_{2}^{-1}(t)\right]\right)=\frac{1}{2 \pi i} \int_{C} \ln ^{2}(z) \tau\left[\left(z-\gamma_{1}(t) \gamma_{2}^{-1}(t)\right)^{-1}\right] d z .
$$

Now we compute $f^{\prime}(0)$; note first that $\gamma_{1}(0) \gamma_{2}^{-1}(0)=p q^{-1}$ and also that

$$
\frac{d}{d t}{ }_{t=0} \gamma_{1}(t) \gamma_{2}^{-1}(t)=-v q^{-1}+p q^{-1} w q^{-1}
$$


Using the properties of the trace we get

$$
\begin{aligned}
& \frac{d}{d t}_{t=0} f(t)=-\frac{1}{2 \pi i} \int_{C} \ln ^{2}(z) \tau\left[\left(z-p q^{-1}\right)^{-2}\left(-v q^{-1}+p q^{-1} w q^{-1}\right)\right] d z= \\
& =\tau\left[\left(-\frac{1}{2 \pi i} \int_{C} \ln ^{2}(z)\left(z-p q^{-1}\right)^{-2} d z\right)\left(-v q^{-1}+p q^{-1} w q^{-1}\right)\right] .
\end{aligned}
$$

If we integrate by parts the first factor inside the trace, we obtain (note that $\frac{d}{d z} \ln ^{2}(z)=2 \ln (z) z^{-1}=2 z^{-1} \ln (z)$ and $C$ is a closed curve) that

$$
\begin{aligned}
\dot{f}(0)= & \tau\left[\left(\frac{1}{2 \pi i} \int_{C} 2 \ln (z) z^{-1}\left(z-p q^{-1}\right)^{-1} d z\right)\left(-v q^{-1}+p q^{-1} w q^{-1}\right)\right]= \\
= & -\tau\left[\left(\frac{1}{2 \pi i} \int_{C} 2 \ln (z) z^{-1}\left(z-p q^{-1}\right)^{-1} d z\right) v q^{-1}\right]+ \\
& +\tau\left[\left(\frac{1}{2 \pi i} \int_{C} 2 \ln (z) z^{-1}\left(z-p q^{-1}\right)^{-1} d z\right) p q^{-1} w q^{-1}\right] .
\end{aligned}
$$

Therefore,

$$
\begin{aligned}
\dot{f}(0)= & -\frac{1}{2 \pi i} \int_{C} 2 \ln (z) z^{-1} \tau\left[q^{-1}\left(z-p q^{-1}\right)^{-1} v\right] d z+ \\
& +\frac{1}{2 \pi i} \int_{C} 2 \ln (z) z^{-1} \tau\left[q^{-\frac{1}{2}}\left(z-p q^{-1}\right)^{-1} p q^{-1} w q^{-\frac{1}{2}}\right] d z .
\end{aligned}
$$

Using the elementary identities

$$
p^{\frac{1}{2}}\left(z-p^{\frac{1}{2}} q^{-1} p^{\frac{1}{2}}\right)^{-1} p^{-\frac{1}{2}}=\left(z-p q^{-1}\right)^{-1}=q^{\frac{1}{2}}\left(z-q^{-\frac{1}{2}} p q^{-\frac{1}{2}}\right)^{-1} q^{-\frac{1}{2}}
$$

one arrives to the expression

$$
\begin{aligned}
\dot{f}(0)= & -\frac{1}{2 \pi i} \int_{C} 2 \ln (z) z^{-1} \tau\left[q^{-1} p^{\frac{1}{2}}\left(z-p^{\frac{1}{2}} q^{-1} p^{\frac{1}{2}}\right)^{-1} p^{-\frac{1}{2}} v\right] d z+ \\
& +\frac{1}{2 \pi i} \int_{C} 2 \ln (z) z^{-1} \tau\left[\left(z-q^{-\frac{1}{2}} p q^{-\frac{1}{2}}\right)^{-1} q^{-\frac{1}{2}} p q^{-\frac{1}{2}} q^{-\frac{1}{2}} w q^{-\frac{1}{2}}\right] d z= \\
= & -2 \tau\left[q^{-1} p^{\frac{1}{2}} p^{-\frac{1}{2}} q p^{-\frac{1}{2}} \ln \left(p^{\frac{1}{2}} q^{-1} p^{\frac{1}{2}}\right) p^{-\frac{1}{2}} v\right]+ \\
& +2 \tau\left[\ln \left(q^{-\frac{1}{2}} p q^{-\frac{1}{2}}\right) q^{\frac{1}{2}} p^{-1} q^{\frac{1}{2}} q^{-\frac{1}{2}} p q^{-\frac{1}{2}} q^{-\frac{1}{2}} w q^{-\frac{1}{2}}\right)= \\
= & -2 \tau\left[\ln \left(p^{\frac{1}{2}} q^{-1} p^{\frac{1}{2}}\right) p^{-\frac{1}{2}} v p^{-\frac{1}{2}}\right]+2 \tau\left[\ln \left(q^{-\frac{1}{2}} p q^{-\frac{1}{2}}\right) q^{-\frac{1}{2}} w q^{-\frac{1}{2}}\right]=0+0=0,
\end{aligned}
$$

which holds by the orthogonality relations (12), naming $x=\ln \left(p^{\frac{1}{2}} q^{-1} p^{\frac{1}{2}}\right)$ (recall that $M$ is convex $)$, and $y=\ln \left(q^{-\frac{1}{2}} p q^{-\frac{1}{2}}\right)$.

Since $f(t)$ is a convex function, $f$ has a global minimum at $t=0$, which proves that $\operatorname{dist}\left(\Pi_{M}(r), \Pi_{M}(s)\right)=\operatorname{dist}(p, q) \leq \operatorname{dist}(r, s)$. 
Lemma 5.3. Let $r \in \Sigma$ and let $M$ be a convex exponential set. The there exists $p \in M$ such that dist $(r, M)=\operatorname{dist}(r, p)$ if and only if there is a geodesic through $r$ orthogonal to $M$.

Proof. Assume first that there is a point $p \in M$ such that the geodesic $\gamma$ through $p$ and $r$ is orthogonal to $M$ at $p$. Now for any point $q \in M$, take a geodesic $\beta$ joining $q$ and $r$, and a geodesic $\delta$ joining $p$ to $q$. Consider the geodesic triangle with sides $\gamma, \beta, \delta$; the angle opposite to $\beta$ is a right angle, so (see Lemma 3.6)

$$
\text { Length }(\beta)^{2} \geq \operatorname{Length}(\delta)^{2}+\operatorname{Length}(\gamma)^{2} \geq \operatorname{Length}(\gamma)^{2} .
$$

This proves that $\operatorname{dist}(p, r) \leq \operatorname{dist}(q, r)$ for any $q \in M$.

Assume now that $p \in M$ has the minimizing property and consider, for any point $q \in M$, the geodesic $\gamma_{p, q}(s)$ joining $p$ to $q$ (note that it is inside $M$ for any $s$ by virtue of the convexity). Now consider the family of geodesics

$$
\gamma_{s}(t)=\gamma_{r, \gamma_{p q}(s)}(t)=r^{\frac{1}{2}}\left(r^{-\frac{1}{2}} p^{\frac{1}{2}}\left(p^{-\frac{1}{2}} q p^{-\frac{1}{2}}\right)^{s} p^{\frac{1}{2}} r^{-\frac{1}{2}}\right)^{t} r^{\frac{1}{2}}
$$

that is, the family of geodesics joining $r$ to $\gamma_{p, q}(s)$.

Put $g(s)=\operatorname{Length}\left(\gamma_{s}\right)^{2}=\operatorname{dist}\left(r, \gamma_{p, q}(s)\right)$. This function has a minimum at $s=0$, hence (since it is $C^{\infty}$ ) it must be that $\dot{g}(0)=0$. As in the proof of the previous theorem, we have

$$
g(s)=\tau\left(\ln ^{2}\left(r \gamma_{p, q}(s)^{-1}\right)\right)=\frac{1}{2 \pi i} \int_{C} \ln ^{2}(z) \tau\left[\left(z-r \gamma_{p, q}^{-1}(s)\right)^{-1}\right] d z .
$$

Taking the derivative at $s=0$ and integrating by parts we obtain

$$
0=\dot{g}(0)=-2 \tau\left(\ln \left(r p^{-1}\right) \ln \left(q p^{-1}\right) p^{-1}\right)=2 \tau\left(\ln \left(q p^{-1}\right) p^{-1} \ln \left(p r^{-1}\right)\right) .
$$

On the other hand, the angle subtended by $\gamma_{p, q}$ and $\gamma_{r, p}$ at $p$ is

$$
<\dot{\gamma}_{r, p}(1), \dot{\gamma}_{p, q}(0)>_{p}=\tau\left(\ln \left(q p^{-1}\right) p^{-1} \ln \left(p r^{-1}\right)\right) .
$$

This proves that $\gamma_{r, p}$ is orthogonal to any geodesic at $p$ contained in $M$, and by definition, it is orthogonal to $M$.

The following is related to the main result in [6] by H. Porta and L. Recht:

Theorem 5.4. If $M=e^{H}$ is a convex exponential set, and there is a closed, orthogonal supplement $S$ for $H$ (namely $\mathcal{A}_{h}=H \oplus_{\perp_{\tau}} S$ ) then for any point $r \in \Sigma$ there is a geodesic through $r$ orthogonal to $M$.

Proof. Exactly as in [6], there is an equality of sets $E(N M)=\Sigma$, where $N M$ stands for the normal bundle of $M$, i.e the pairs $(p, v)$ with $p \in M$ and $v \perp_{p} M$. 
The typical examples for this situation arise when $H=B_{h}$ for a subalgebra $B$ of $\mathcal{A}$. In this case, by a result of Takesaki [8], there is a conditional expectation $\mathcal{E}: \mathcal{A} \rightarrow \mathcal{A}$ with $\operatorname{rank} B$, compatible with $\tau$ (i.e $\tau(\mathcal{E}(x))=\tau(x)$ for any $x \in \mathcal{A})$.

Corollary 5.5. If $H$ is a closed subspace in $\mathcal{A}_{h}$ (supplemented as in the previous theorem) such that $[x,[x, y]] \in H$ whenever $x, y \in H$, then for any $z \in \mathcal{A}_{h}$ we can factor

$$
e^{z}=e^{y} e^{w} e^{y}
$$

for unique $y \in H$ and $w \in \mathcal{A}_{h}$ such that $\tau(w x)=0$ for any $x \in H$. Moreover, $e^{2 y}$ minimizes (geodesic) distance between $M=e^{H}$ and $e^{z}$, and is unique with that property.

Corollary 5.6. Fix $\mathcal{D}$ a m.a.s.a of $\mathcal{A}$. Then for any $x \in \mathcal{A}_{h}$ there are unique $d \in \mathcal{D}_{h}$ and $v \in \mathcal{A}_{h}$ such that $\tau(v z)=0$ for any $z \in \mathcal{D}$, and $e^{x}=d e^{v} d$

Corollary 5.7. If $H$ is a closed, supplemented subspace in $\mathcal{A}_{h}$ such that $[x,[x, y]] \in$ $H$ whenever $x, y \in H$, then for any $g \in G_{\mathcal{A}}$ we can factor

$$
g=e^{x} e^{y} u
$$

for unique $x \in H, y \in H^{\perp}$ and $u$ in the unitary group of $\mathcal{A}$.

Proof. Note that $g g^{*} \in \Sigma$ hence $g g^{*}=e^{x} e^{2 y} e^{x}$ where $x, y$ are as required. Now take $u=e^{-y} e^{-x} g$; a straightforward computation shows that $u u^{*}=u^{*} u=1$. Uniqueness follows from the uniqueness of $x, y$.

\section{References}

[1] R. Bhatia, On the exponential metric increasing property. Linear Algebra Appl. 375 (2003) 211-220.

[2] G. Corach, H. Porta, L. Recht, The geometry of the space of selfadjoint invertible elements in a C*-algebra. Integral Equations Operator Theory 19 (1993) 333-359.

[3] G. Corach, H. Porta, L. Recht, Convexity of the geodesic distance on spaces of positive operators. Illinois J. Math. 38 (1994) 87-94.

[4] P. Eberlein, Structure of manifolds of nonpositive curvature. Global differential geometry and global analysis. Lecture Notes in Mathematics 1156, Springer, Berlin, 1985, 86-153.

[5] G. D. Mostow, Some new decomposition theorems for semi-simple groups. Mem. Amer. Math. Soc. 14 (1955) 31-54.

[6] H. Porta, L. Recht, Conditional expectations and operator decompositions. Ann. Global Anal. Geom. 12 (1994) 335-339. 
[7] H. Porta, L. Recht, Exponential sets an their geometric motions. J. Geom. Anal. (2) 6 (1996) 277-285.

[8] M. Takesaki, Conditional Expectations in a von Neumann algebra. J. Funct. Anal. 9 (1972) 306-321.

Esteban Andruchow and Gabriel Larotonda

Instituto de Ciencias

Universidad Nacional de Gral. Sarmiento

J. M. Gutierrez 1150

(1613) Los Polvorines

Argentina

e-mail: eandruch@ungs.edu.ar, glaroton@ungs.edu.ar 